UDC 621.311

SCOPUS CODE 2105

https://doi.org/10.36073/1512-0996-2021-3-76-82

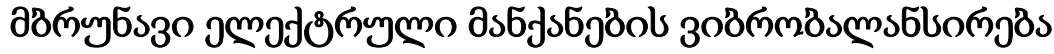

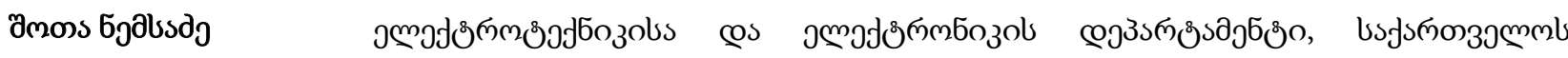

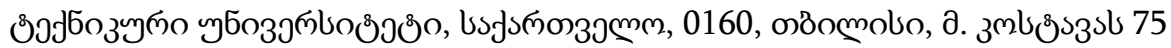 \\ E-mail: sh-nemsadze@mail.ru

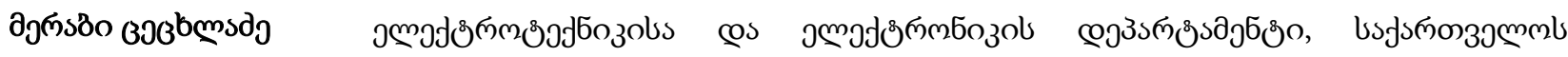

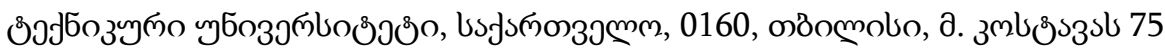 \\ E-mail: merabi.tsetskhladze@mail.ru

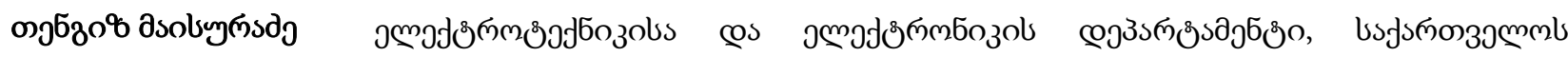

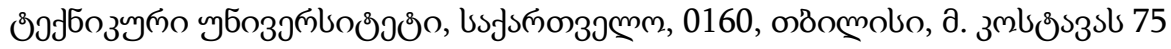 \\ E-mail: tengizim@gmail.com
}

\section{๓ว}

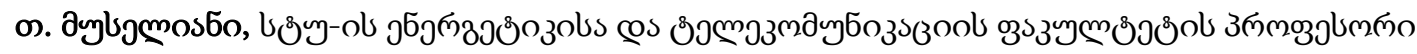

E-mail: museliani@yahoo.com

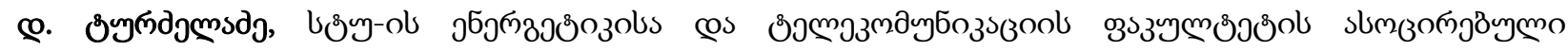

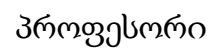

E-mail: turdzeladze59@gmail.com

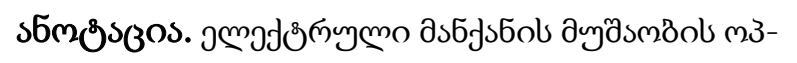

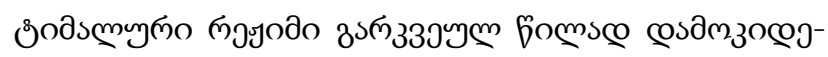

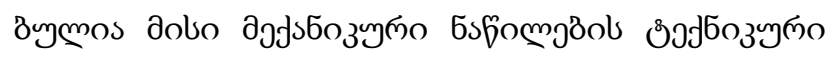

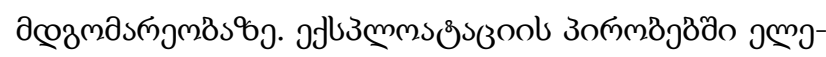

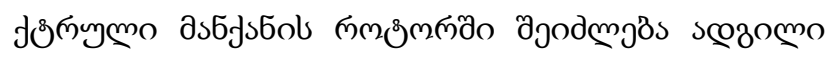

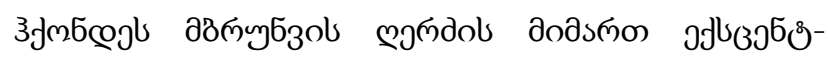

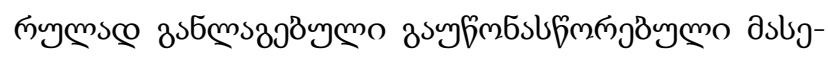

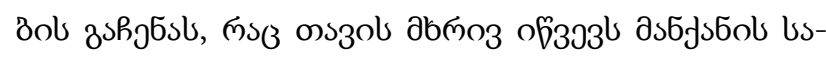

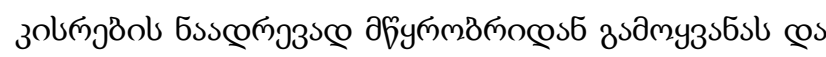

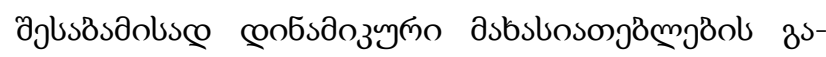

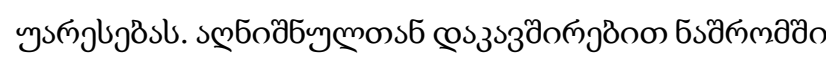

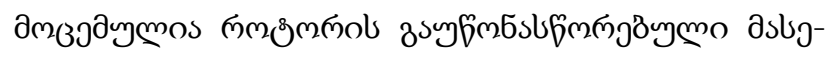

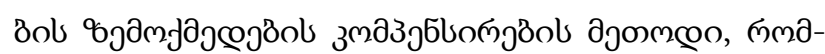

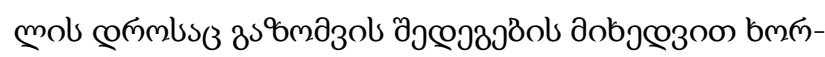

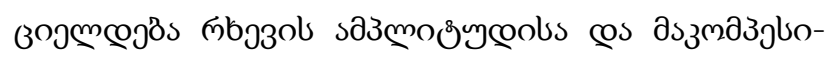

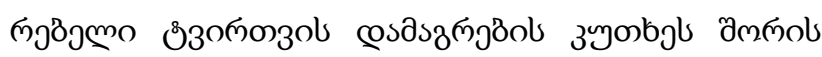

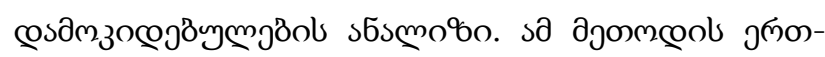

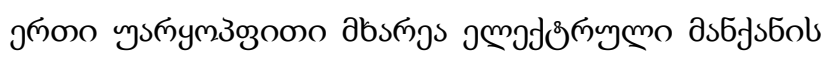

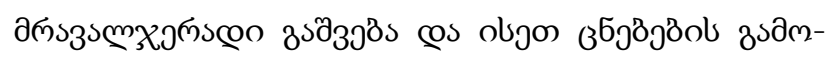

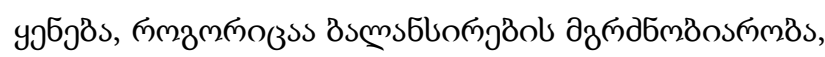

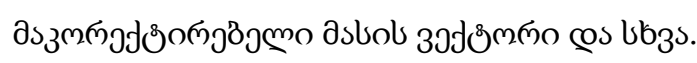

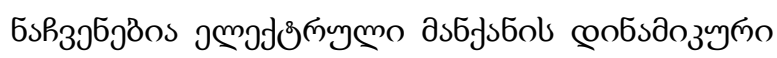

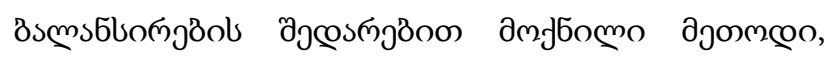




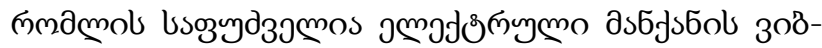

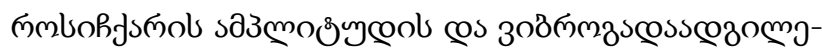

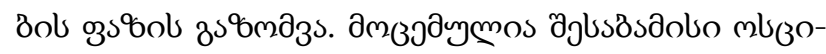

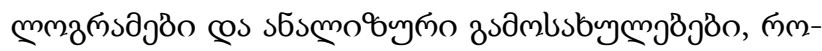

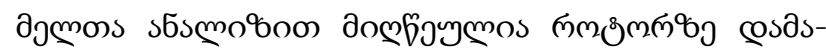

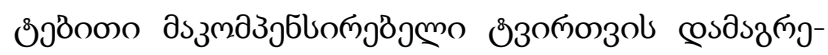

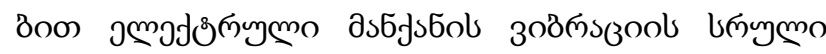
sм3зэos.

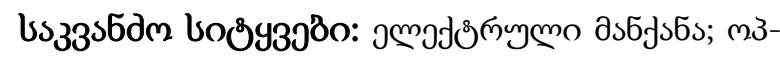

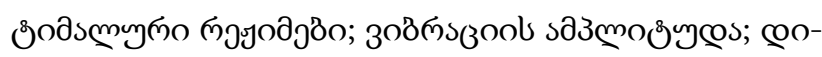

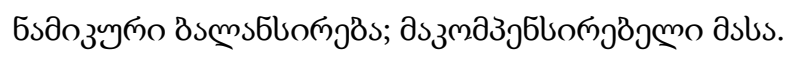

\section{yjuszsmo}

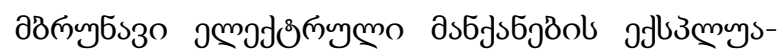

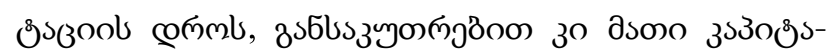

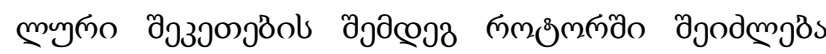

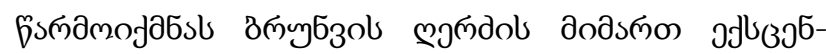

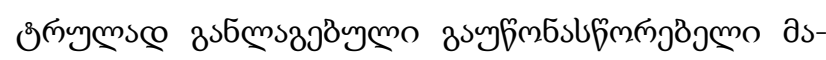

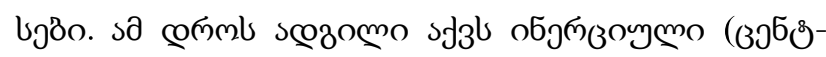

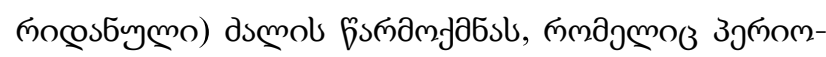

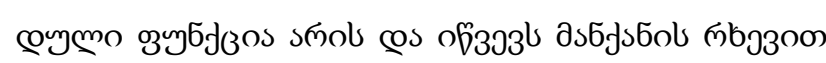

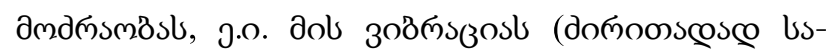

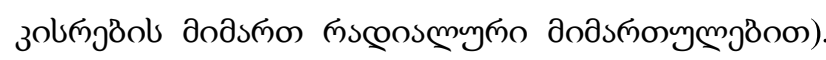

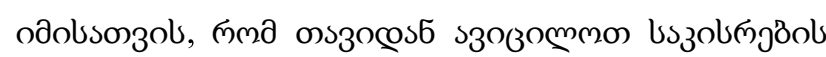

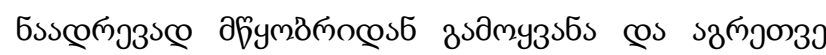

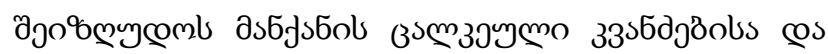

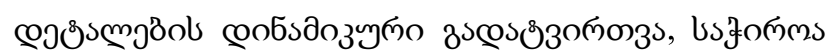

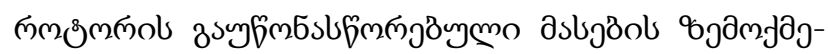

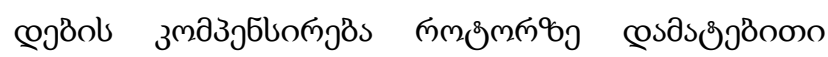

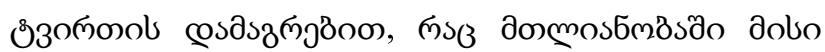

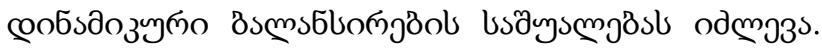

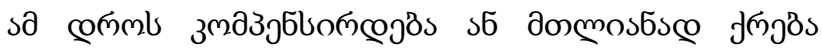

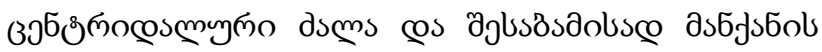

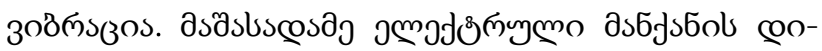

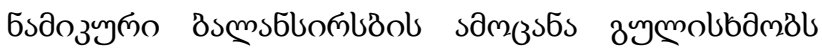

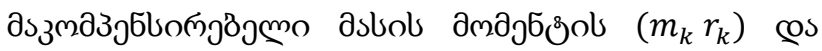

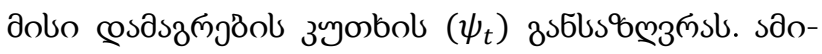

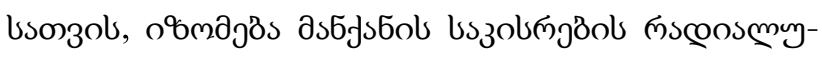

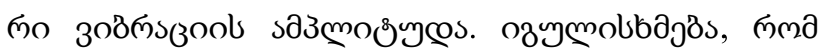

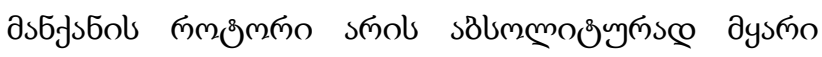

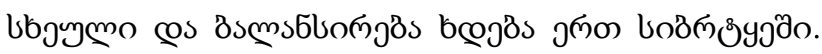

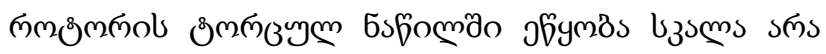

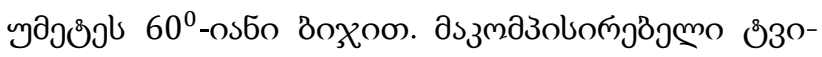

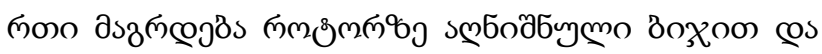

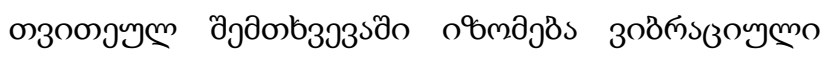

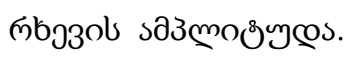

\section{donoossco bsfomo}

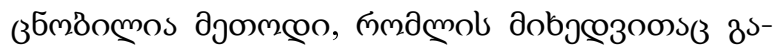

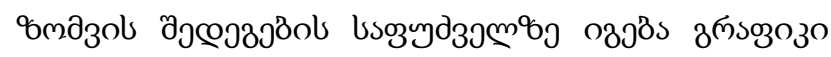

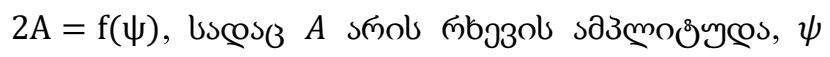

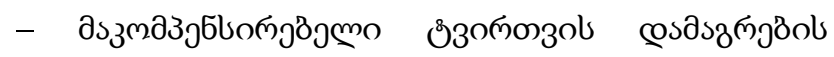

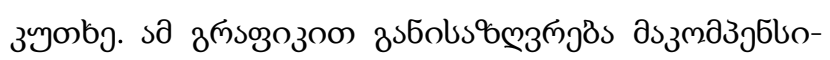

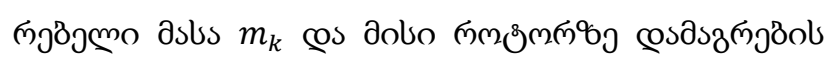

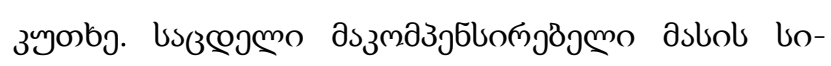

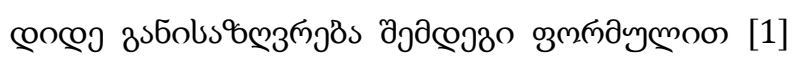

$$
\mathrm{m}_{\mathrm{b}_{3}}=(0,1-0,2) \frac{\mathrm{Gg}}{\omega^{2} \mathrm{r}}(1)
$$

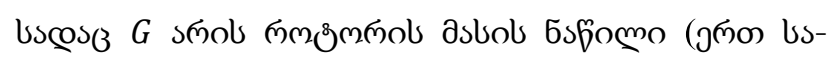

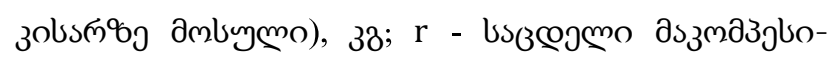

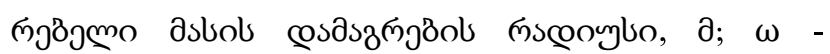

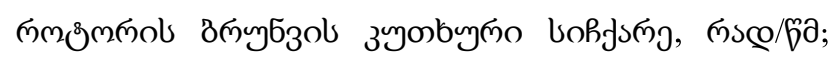
$g=9,81 \partial / 6 \partial^{2}$. 


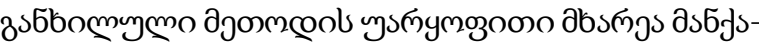

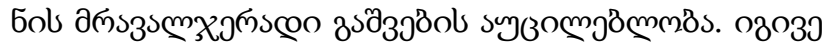

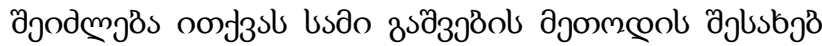

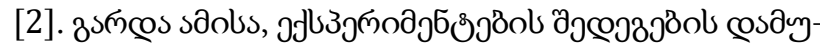

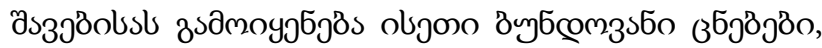

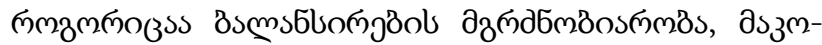

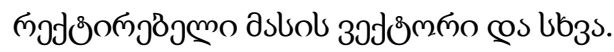

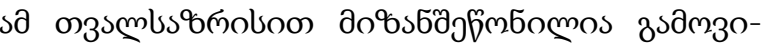

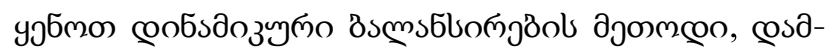

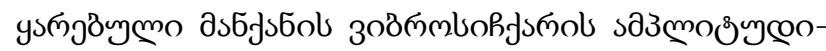

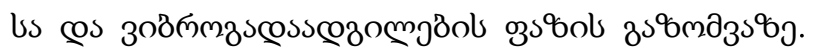

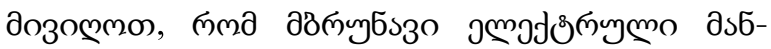

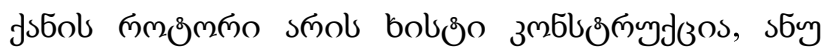

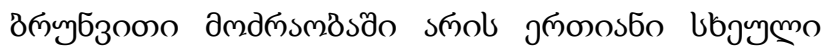

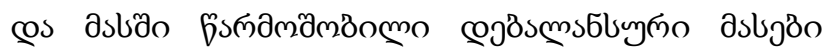

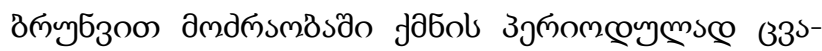

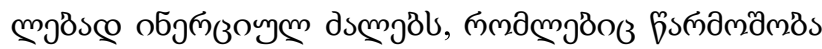

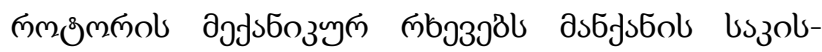

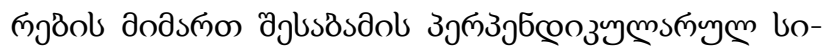

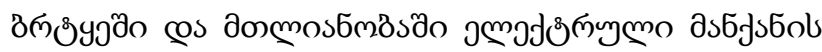

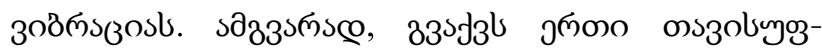

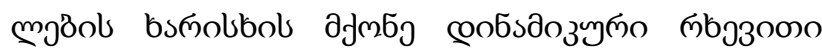

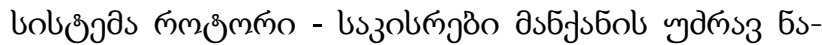

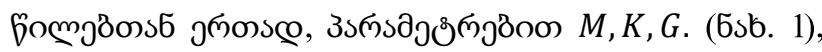

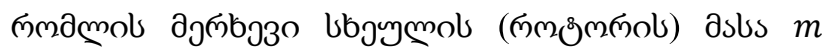

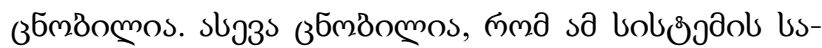

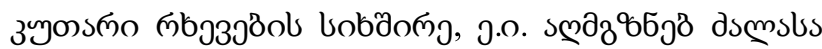

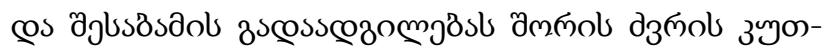
by smol cosзs\%mbةo $0<\varphi<90^{\circ}$.

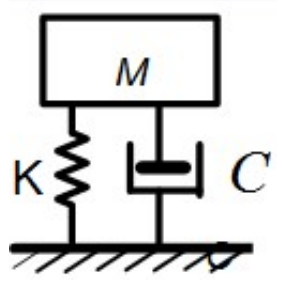

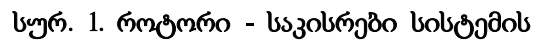

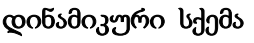

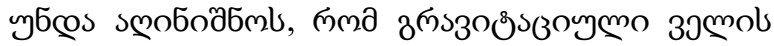

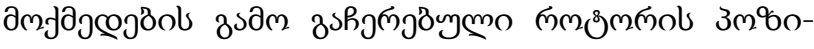

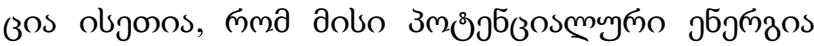

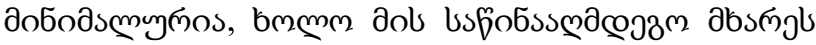

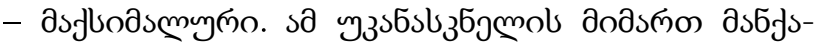

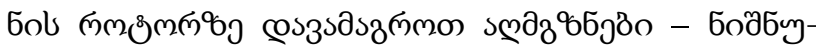

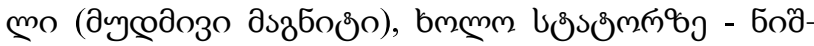

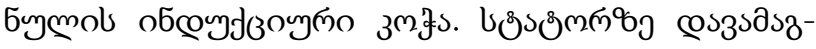

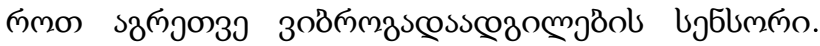

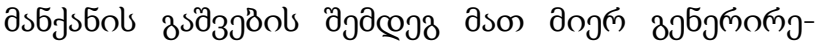

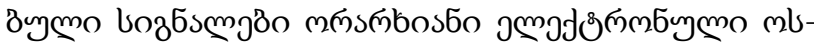

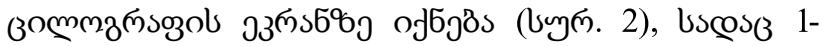

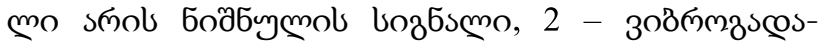

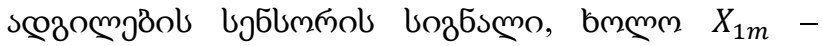

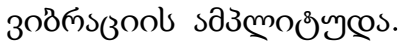

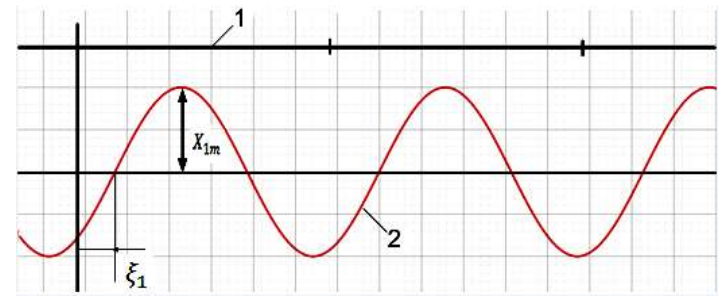

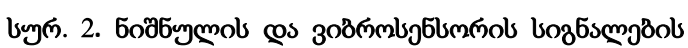
mbुommgnsas 


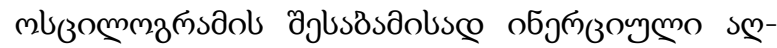

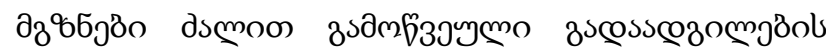

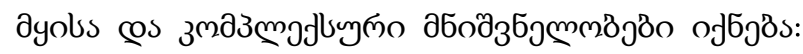

$$
X_{1}(t)=X_{1 m} \sin \left(\omega t-\xi_{1}\right) \Rightarrow \dot{X}_{1 m}=X_{1 m} e^{-j \xi_{1}},
$$

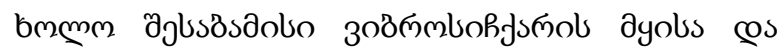

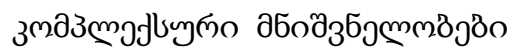

$$
\begin{gathered}
v_{1}(t)=V_{1 m} \sin \left(\omega t+\frac{\pi}{2}-\xi_{1}\right) \Rightarrow \dot{V}_{1 m}= \\
V_{1 m} e^{j\left(\frac{\pi}{2}-\xi_{1}\right)}
\end{gathered}
$$

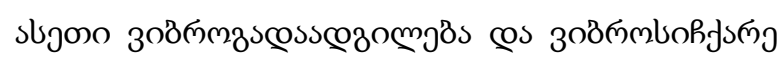

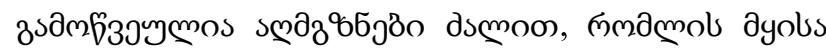

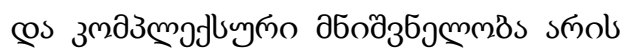

$$
f_{1}(t)=F_{1 m} \sin \left(\omega t+\psi_{1}\right) \Rightarrow \dot{F}_{1 m}=F_{1 m} e^{j \psi_{1}},
$$

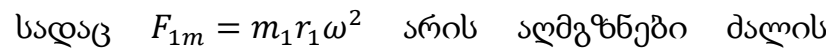

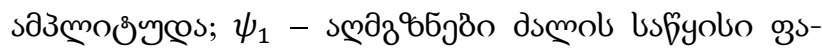

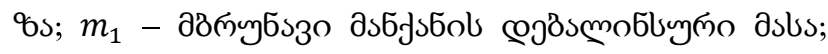

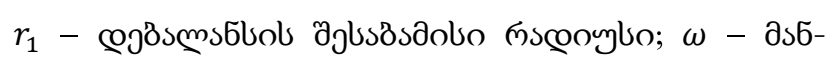

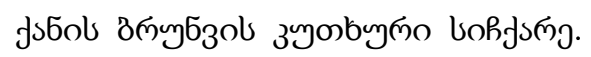

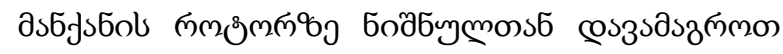

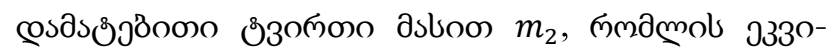

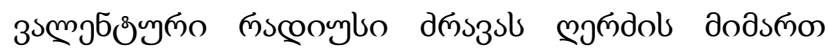

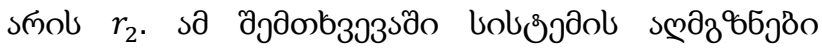

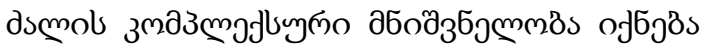

$$
F^{\prime}{ }_{1 m}=\dot{F}_{1 m}+\dot{F}_{2 m},
$$

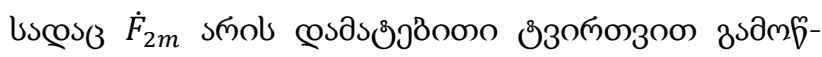

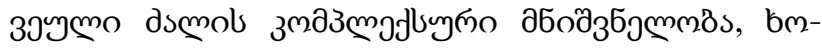

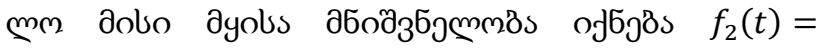
$m_{2} r_{2} \omega^{2} \sin \left(\omega t+\psi_{2}\right)=m_{2} r_{2} \omega^{2} \sin (\omega t), \quad$ пзсозбьз

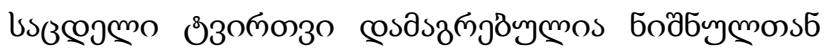

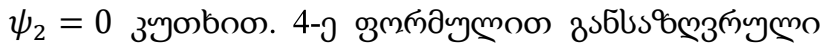

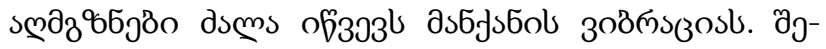

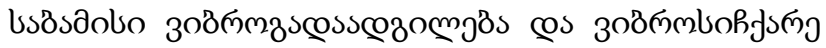
ofbวðัs

$$
\begin{aligned}
& X_{1}(t)=X_{1 m} \sin \left(\omega t-\xi_{2}\right)=X_{1}(t)+X_{2}(t) . \\
& v_{1}(t)=V_{1 m} \sin \left(\omega t+\frac{\pi}{2}-\xi_{2}\right)=v_{1}(t)+v_{2}(t) \text {. }
\end{aligned}
$$

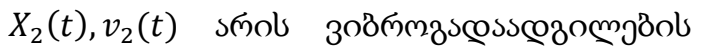

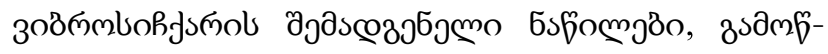

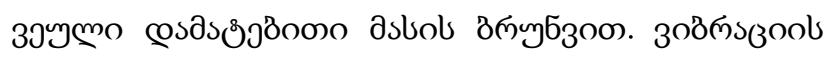

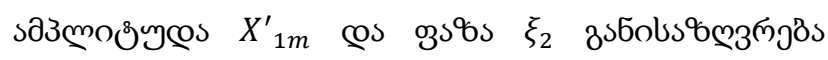

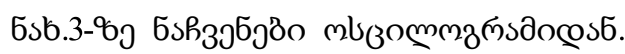

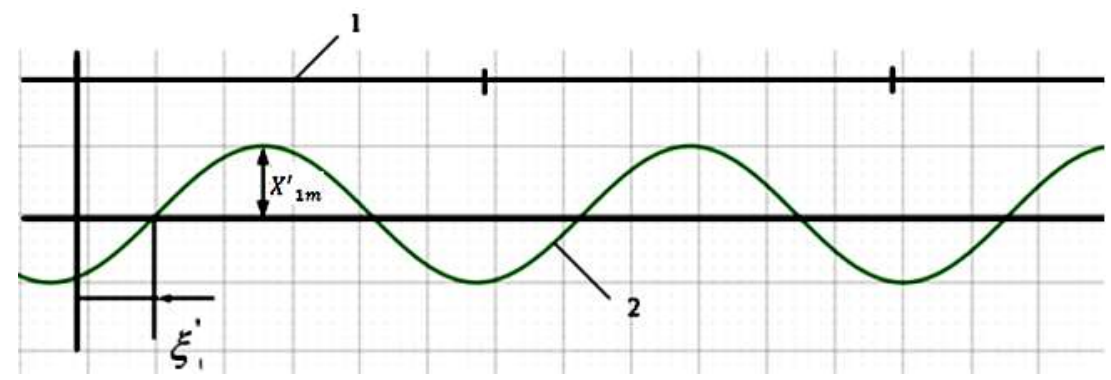

uym. 3. asб

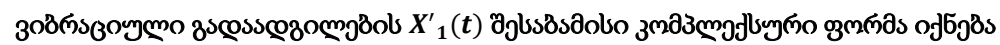

$$
\dot{X}^{\prime}{ }_{1 m}=X^{\prime}{ }_{1 m} e^{-j \xi_{2}}
$$

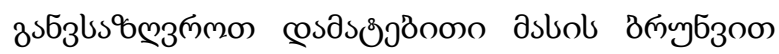

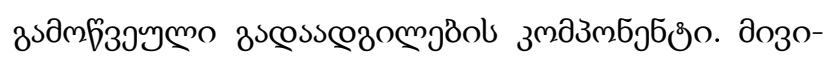

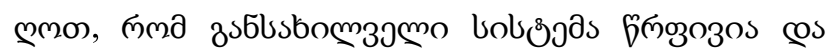

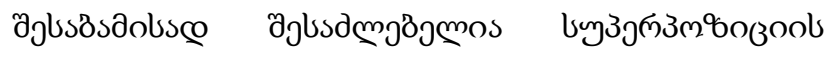

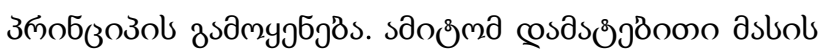

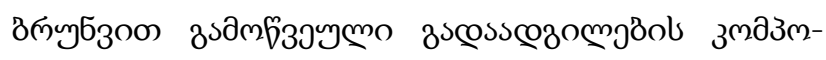

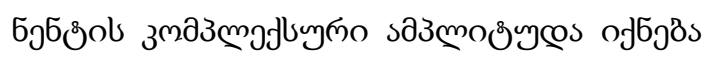




$$
\begin{gathered}
\dot{X}_{2 m}=\dot{X}^{\prime}{ }_{1 m}-\dot{X}_{1 m}=X^{\prime}{ }_{1 m} e^{-j \xi_{2}}-X_{1 m} e^{-j \xi_{1}}= \\
X^{\prime}{ }_{1 m}\left(\cos \xi_{2}-j \sin \xi_{2}\right)-X_{1 m}\left(\cos \xi_{1}-j \sin \xi_{1}\right)= \\
\left(X^{\prime}{ }_{1 m} \cos \xi_{2}-X_{1 m} \cos \xi_{1}\right)- \\
-j\left(X^{\prime}{ }_{1 m} \sin \xi_{2}-X_{1 m} \sin \xi_{1}\right)= \\
=X_{2 m} e^{-j \xi_{2}}
\end{gathered}
$$

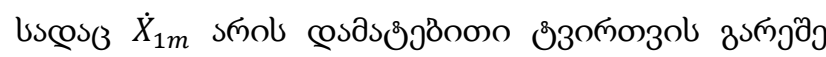

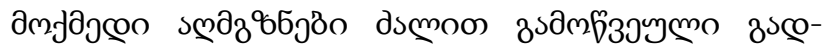

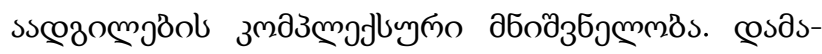

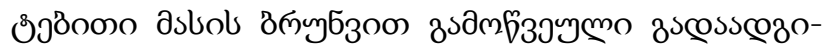

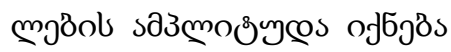

$$
\begin{gathered}
X_{2 m}= \\
\sqrt{\left(X^{\prime}{ }_{1 m} \cos \xi_{2}^{\prime}-X_{1 m} \cos \xi_{1}\right)^{2}+\left(X^{\prime}{ }_{1 m} \sin \xi_{2}^{\prime}-X_{1 m} \sin \xi_{1}\right)^{2}},
\end{gathered}
$$

bмmल оुsकs

$$
\xi_{2}=\operatorname{arctg} \frac{X^{\prime}{ }_{1 m} \sin \xi_{2}^{\prime}-X_{1 m} \sin \xi_{1}}{X^{\prime}{ }_{1 m} \cos \xi^{\prime}{ }_{2}-X_{1 m} \cos \xi_{1}} .
$$

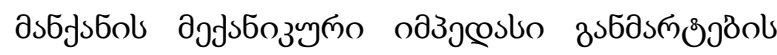

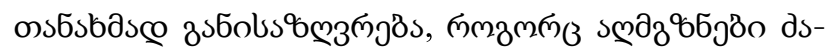

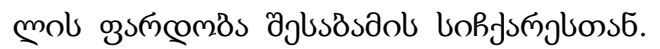

$$
\begin{aligned}
\underline{Z}_{1 \mathrm{~m}}=\frac{\dot{\mathrm{F}}_{1 \mathrm{~m}}}{\dot{\mathrm{V}}_{1 \mathrm{~m}}}= & \frac{\dot{\mathrm{F}}_{2 \mathrm{~m}}}{\dot{\mathrm{V}}_{2 \mathrm{~m}}}=\frac{\dot{\mathrm{F}}^{\prime}{ }^{\prime} \mathrm{m}}{\mathrm{V}^{\prime}{ }_{2 \mathrm{~m}}}=\frac{\mathrm{F}^{\prime}{ }_{2 \mathrm{~m}} \mathrm{e}^{\mathrm{j} 0^{0}}}{\mathrm{~V}^{\prime}{ }_{2 \mathrm{~m}} \mathrm{e}^{-\mathrm{j}\left(\frac{\pi}{2}-\xi_{2}\right)}}= \\
& \frac{\mathrm{F}_{2 \mathrm{~m}}}{\mathrm{X}_{2 \mathrm{~m}} \omega} \mathrm{e}^{\mathrm{j}\left(\xi_{2}-\frac{\pi}{2}\right)} .
\end{aligned}
$$

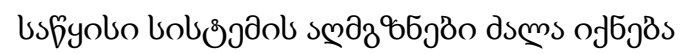

$$
\begin{aligned}
\dot{\mathrm{F}}_{1 \mathrm{~m}}= & \dot{\mathrm{V}}_{1 \mathrm{~m}} \underline{\mathrm{Z}}_{1 \mathrm{~m}}=\mathrm{X}_{1 \mathrm{~m}} \omega \mathrm{e}^{\mathrm{j}\left(\frac{\pi}{2}-\xi_{1}\right)}\left(\frac{\mathrm{F}_{2 \mathrm{~m}}}{\mathrm{X}_{2 \mathrm{~m}} \omega} \mathrm{e}^{\mathrm{j}\left(\xi_{2}-\frac{\pi}{2}\right)}\right)= \\
& \frac{\mathrm{X}_{1 \mathrm{~m}}}{\mathrm{X}_{2 \mathrm{~m}}} \mathrm{~m}_{2} \mathrm{r}_{2} \omega^{2} \mathrm{e}^{\mathrm{j}\left(\xi_{2}-\xi_{1}\right)}=\mathrm{F}_{1 \mathrm{~m}} e^{j \Psi_{1}}
\end{aligned}
$$

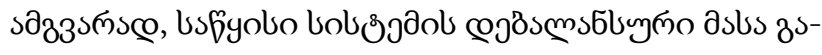

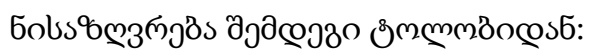

$$
\frac{X_{1 m}}{X_{2 m}} m_{2} r_{2} \omega^{2}=m_{1} m_{1} \omega^{2} \Rightarrow m_{1}=m_{2} \frac{r_{2} X_{1 m}}{r_{1} X_{2 m}}
$$

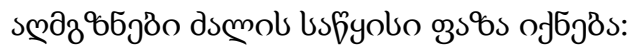

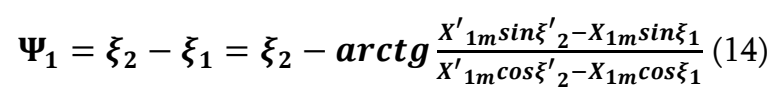

\section{cosb $33^{5 s}$}

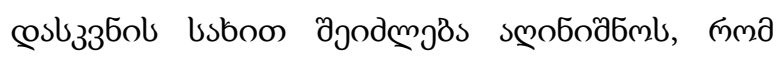

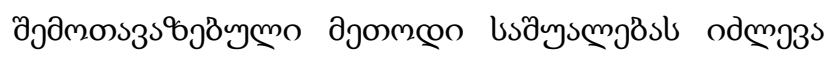

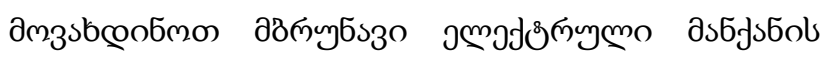

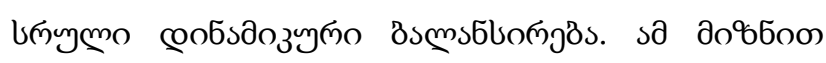

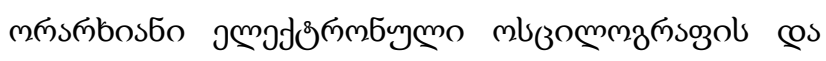

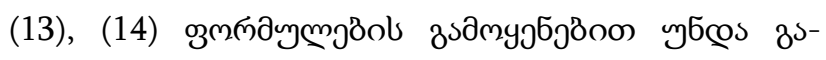

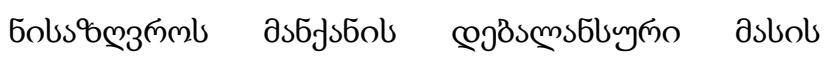

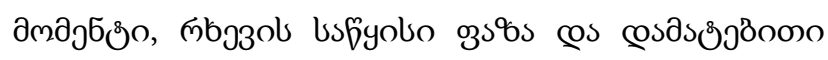

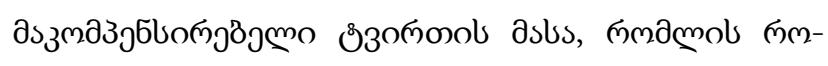

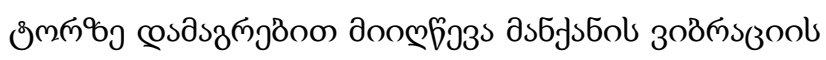

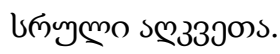

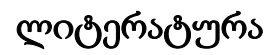

1. Rivlin, L. B. (1956). Installation of large electrical machines. Leningrad: Energy. (In Russian);

2. Gemke, R.G. (1989). Malfunctions of electrical machines. Leningrad: Energy. (In Russian);

3. Iorish, Yu., Vibrometry, I. (1963). Mechanical engineering. Leningrad: Energy. (In Russian);

4. Brown, M., Routani, J., Petyl, D. (2007). Diagnostics and troubleshooting of electrical equipment and control circuits. Moscow: Dodeka-XXI. (In Russian). 
UDC 621.311

SCOPUS CODE 2105

https://doi.org/10.36073/1512-0996-2021-3-76-82

\title{
Vibro Balancing of Rotating Electric Vehicles
}

\author{
Shota Nemsadze Department of Electrical Engineering and Electronics, Georgian Technical University, \\ Georgia, 0160, Tbilisi, 75 M. Kostava str. \\ E-mail: sh-nemsadze@mail.ru \\ Merab Tsetskhladze Department of Electrical Engineering and Electronics, Georgian Technical University, \\ Georgia, 0160, Tbilisi, 75 M. Kostava str. \\ E-mail: merabi.tsetskhladze@mail.ru \\ Tengiz Maisuradze Department of Electrical Engineering and Electronics, Georgian Technical University, \\ Georgia, 0160, Tbilisi, 75 M. Kostava str. \\ E-mail: tengizim@gmail.com
}

\section{Reviewers:}

T. Museliani, Professor, Faculty of Energy and Telecommunications, GTU

E-mail: museliani@yahoo.com

D. Turdzeladze, Associate Professor, Faculty of Energy and Telecommunications, GTU

E-mail: turdzeladze59@gmail.com

Abstract. The optimal mode of operation of an electric car depends to some extent on the technical condition of its mechanical parts. Under operating conditions, unbalanced masses, eccentrically located in relation to the axis of rotation, may occur in the rotor of an electric car, which, in turn, will lead to premature output of machine bearings and, as a consequence, to a deterioration in dynamic characteristics. In this regard, the paper presents a method of compensating for the impact of unbalanced masses of the rotor, during which the analysis of the relationship between the amplitude of the oscillation and the angle of attachment of the compensating load is performed according to the measurement results. One of the downsides of this method is the multiple launches of the electric car and the use of concepts such as balancing sensitivity, corrective mass and more.

A relatively flexible method of dynamic balancing of an electric car is shown, based on the measurement of the vibration speed amplitude and vibration phase of the electric car. Relevant oscillograms and analytical images are given, the analysis of which achieves complete suppression of electric vehicle vibration by attaching additional compensating load to the rotor.

Key words: balancing; compensating mass; electric car; optimal modes; vibration amplitude.

ISSN 1512-0996

www.shromebi.gtu.ge 


\title{
UDC 621.311
}

SCOPUS CODE 2105

https://doi.org/10.36073/1512-0996-2021-3-76-82

\section{Вибробалансировка вращающихся электромобилей}

\author{
Шота Немсадзе Департамент электротехники и электроники, Грузинский технический \\ университет, Грузия, 0160, Тбилиси, ул. М. Костава 75 \\ E-mail: sh-nemsadze@mail.ru \\ Мераб Цецхладзе Департамент электротехники и электроники, Грузинский технический \\ университет, Грузия, 0160, Тбилиси, ул. М. Костава 75 \\ E-mail: merabi.tsetskhladze@mail.ru \\ Тенгиз Маисурадзе Департамент электротехники и электроники, Грузинский технический \\ университет, Грузия, 0160, Тбилиси, ул. М. Костава 75 \\ E-mail: tengizim@gmail.com
}

\section{Рецензенты:}

Т. Муселиани, профессор факультета энергетики и телекоммуникаций ГТУ

E-mail: museliani@yahoo.com

Д. Турдзеладзе, ассоциированный профессор факультета энергетики и телекоммуникаций ГТУ

E-mail: turdzeladze59@gmail.com

Аннотация. Оптимальный режим работы электромашин в определенной степени зависит от технического состояния его механических частей. В рабочих условиях несбалансированная масса, эксцентрично расположенная относительно оси вращения, может возникнуть в роторе электромашин, что, в свою очередь, приведет к преждевременному выходу подшипников машин и, как следствие, к ухудшению динамических характеристик. В связи с этим в статье представлен метод компенсации воздействия неуравновешенных масс на ротор, при котором по результатам измерений проводится анализ зависимости между амплитудой колебаний и углом приложения компенсирующей нагрузки. Один из недостатков этого метода - многократные запуски электромашин и использование таких понятий, как: балансировочная чувствительность, корректирующий вектор массы и многое другое.

Показан относительно гибкий метод динамической балансировки электромашин, основанный на измерении амплитуды, скорости и фазы вибрации электромашин. Приведены соответствующие осциллограммы и аналитические изображения, при анализе которых достигается полное подавление вибрации электромашин путем приложения к ротору дополнительной компенсирующей нагрузки.

Ключевые слова: амплитуда вибрации; балансировка; компенсирующая масса; оптимальные режимы; электромашина.

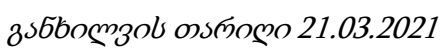

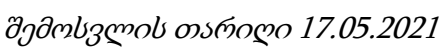

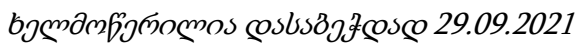

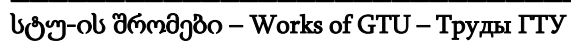

ISSN 1512-0996 